\title{
A Universal Model for Hallucinations and Its Implications
}

\section{Wenge Huang}

\section{Purpose and Method}

- Due to the difficulty in approaching subjective experiences through positive research, the lack of a systematic analytical framework has seriously impeded the in-depth research on hallucinations.

- Different from most research focusing on the generation mechanism of hallucinations from the perspective of neurophysiology, this paper attempts to propose a universal model for hallucinations to reveal the formation mechanism of different states of hallucinations from a unique perspective.

\section{The Inconsistence of the Five Senses}

- Out-of-body Experience: the inconsistence of vision and touching

- Synesthesia: the mismatch of the two pairs of five senses

- Psychedelic phenomena: the integration (not simple superposition) of internal and external stimulations, combined with the amplifying function of enhanced awareness caused by the weakening of the brainfiltering function which always occurs along with

When internal false stimulations and external objective stimulations affect perception together, the changes in their relative strength will result in the consistence, breakdown and re-consistence of the five senses, leading to three basic states of hallucinations:

- "Elementary hallucinations": one can distinguish reality from fantasy

- "Deranged hallucinations": one cannot distinguish reality from fantasy

- "Complete hallucinations": reality and fantasy are totally reversed

\section{A Novel Hypothesis of ESP}

- The essence of Extraordinary Perception (ESP) is that false internal stimulations are mistaken as external objective stimulations which enter through various senses, while real external objective stimulations are mistaken as perceptions which do not result from various senses when one is in "complete hallucinations".

In this condition, one would naturally treat the inconsistent and "unexplainable" external stimulation as information acquired through psychic abilities such as clairaudience, clairvoyance or telepathy since he cannot find the counterpart in the imaginary world. 